\title{
Wesentliche Bereiche des Lehrplans Geographie und Wirt- schaftskunde als Beurteilungsgrundlage
}

\author{
*stefan_hinsch@yahoo.de, Abendgymnasium Wien \& Universität Wien \\ *** herbert.pichler@univie.ac.at, Schwerpunkt Fachdidaktik Geographie und Wirtschaftskunde, Universität Wien \& Schulzentrum Ungargasse \\ $(\triangle$ korresp. Autor) \\ thomas.jekel@sbg.ac.at, Fachbereich Geographie und Geologie, Universität Salzburg \& School of Education, Universität Salzburg
}

Im Sommer 2017 erging an die Arbeitsgruppe für den Lehrplan Geographie und Wirtschaftskunde der Sekundarstufe II AHS der Auftrag, diesen Lehrplan in wesentliche Bereiche zu untergliedern, die als Grundlage der Beurteilung einzelner Teile des semestrierten Lehrplans dienen sollten. Die Begründung dafür lautet wie folgt:

„Mit der Einführung von Semesterprüfungen im Rahmen der Neuen Oberstufe (NOST) stehen bestimmte Aspekte der Leistungsbeurteilung wieder im Fokus. Bei einer negativen Semester-Beurteilung in einem Unterrichtsgegenstand sind im Beiblatt zum Semesterzeugnis (\$22a, Abs. 5, SchUG) jene, wesentlichen Bereiche' der lehrplanmäßigen Anforderungen anzuführen, die nicht ,überwiegend' erfüllt wurden (\$14, Abs. 6, LBVO) und damit den Prüfungsstoff für eine allfällige Semesterprüfung bilden (\$23a, Abs. 5, SchUG)“

(BMB 2017: 1)

Die Arbeitsgruppe stand damit vor der Aufgabe, die wesentlichen Bereiche des Lehrplans 2016 so zu definieren, dass sie den Intentionen (fachliche Stärkung bei gleichzeitiger Aufrechterhaltung der Freiheit der Lehrenden, den Interessen der Schüler/innen angepasst thematisch zu unterrichten, vgl. Jekel \& Pichler 2017) nicht zuwiderlaufen. Gleichzeitig war darauf zu achten, eine Administrierbarkeit auf Seiten der Lehrenden sowie eine Nachvollziehbarkeit für die Schüler/innen sicher zu stellen.

Der vorliegende Text ist ein Vorschlag, der schulintern angepasst werden kann, bei schulautonomen Stundentafeln angepasst werden muss. Er dient als Grundlage der textlichen Hinterlegung im Verwaltungsprogramm Sokrates.

Wie schon bei der Genese des Lehrplans selbst (vgl. Hinsch et al. 2014) wurden Entwürfe sowohl im Rahmen der IMST-Tagung (September 2017) in Klagenfurt diskutiert sowie in einer gemeinsamen Sitzung mit der Bundes-ARGE GW am 6.10.2017 in Strobl akkordiert, sodass von einem praktikablen Instrument ausgegangen werden darf. Der hier dargestellte Vorschlag ist jener, der von beauftragten Arbeitsgruppe an das Ministerium weitergeleitet wurde.

\section{Erläuterungen}

Im vorliegenden Vorschlag wurden in erster Linie die Kompetenzbereiche des semestrierten Lehrplans („Lehrstoff“) als wesentliche Bereiche des Lehrplans ausgewiesen. In ihrer Struktur entsprechen sie weitgehend den Forderungen des Bundesministeriums für Bildung (vgl. BMB 2017), das eine Zahl von 2 bis 5 wesentlichen Bereichen pro Kompetenzmodul vorschlägt.

In einigen Bereichen wurde dennoch, wo inhaltlich möglich, eine vorsichtige Clusterung durchgeführt. So wurden im Kompetenzmodul 6.1 drei kompetenzorientierte Lernziele zu zwei wesentlichen Bereichen zusammengefasst, ebenso im Modul 7.1 und im Modul 8.1. Diese Zusammenfassung ist didaktisch und durch die Erfordernisse der Leistungsbeurteilung begründet: Bei zwei Semesterstunden, in der 6. Klasse lediglich einer Semesterstunde, scheint eine Begrenzung der wesentlichen Bereiche sinnvoll. Ein kompetenzorientierter Unterricht erfordert eine vertiefte Beschäftigung mit Unterrichtsthemen, wodurch bei begrenzter Stundenanzahl größere Themen, die mehrere Lehrplanziele abdecken, wünschenswert sind. Eine zu hohe Anzahl von wesentlichen Bereichen, für die jeweils einzeln eine valide Leistungsfeststellung zu treffen ist, eröffnet die Gefahr eines Abarbeitens von Inhalten, bei dem das Analysieren oder Beurteilen zu kurz kommt. Das würde der Intention des kompetenzorientierten Lehrplans fundamental widersprechen.

Das Kompetenzmodul 8.2 weist zwar im Wirtschaftskundlichen Realgymnasium zwei wesentliche Bereiche auf, in allen anderen Zweigen aber nur einen wesentlichen Bereich. Dies scheint notwendig, 
um den Kompetenzbereich ,Städte als Lebensräume und ökonomische Zentren untersuchen " nicht in zwei wesentliche Bereiche zu zerreißen. Es ist kaum argumentierbar, dass zwischen unterschiedlichen Unterrichtsthemen zu Stadt und Urbanisierung für die Schüler/innen keine Kompensationsmöglichkeit bei der Leistungsbeurteilung bestehen soll.

\section{Empfehlungen für schulautonome Lehrpläne}

Der vorliegende Vorschlag geht von einem Standardlehrplan ohne schulautonome Regelungen aus. Bei schulautonomen Regelungen wären folgende Überlegungen sowohl aus Gründen der Information in Richtung der Schüler/innen als auch aus Gründen der Administrierbarkeit zu beachten:
1. Es erscheint sinnvoll, pro Semester keinesfalls mehr als drei wesentliche Bereiche anzuführen, um die jeweiligen Bereiche mit ausreichend Unterrichtszeit auszustatten.

2. Aus diesem Grund kann es gerade bei schulautonomen Lehrplänen notwendig sein, über die vorgeschlagene Clusterung hinaus einzelne, sachlich gerechtfertigt erscheinende Zusammenfassungen von Kompetenzbereichen des Lehrplans $\mathrm{zu}$ einem wesentlichen Bereich durchzuführen. Allerdings ist hinsichtlich eines kontinuierlichen Kompetenzaufbaus darauf zu achten, dass in den Semestern möglichst eine Gleichgewichtung der Bereiche geographischer und ökonomischer Bildung erhalten bleibt.

\title{
Bildungs- und Lehraufgabe, Lehrstoff
}

\author{
5. Klasse (1. und 2. Semester)
}

Wesentlicher Bereich 1: Gliederungsprinzipien der Erde in unterschiedlichen Sichtweisen

Gliederungsprinzipien der Erde nach unterschiedlichen Sichtweisen reflektieren

- Gliederungsmöglichkeiten der Erde nach naturräumlichen, kulturellen, politischen und ökonomischen Merkmalen analysieren

- Interessensgebundenheit von Gliederungen vergleichen

- Geographien durch Zonierungen/Gliederungen/Grenzziehungen machen und reflektieren

\section{Wesentlicher Bereich 2: Geoökosysteme der Erde}

Geoökosysteme der Erde analysieren

- Klimadaten in Diagramme umsetzen

- Klimagliederungen der Erde vergleichen und hinterfragen

- Wechselwirkungen von Klima, Relief, Boden, Wasser und Vegetation analysieren

- Geoökosysteme und deren anthropogene Überformung erklären

Wesentlicher Bereich 3: Bevölkerung und Gesellschaft

Bevölkerung und Gesellschaft analysieren

- Die heutige und die mögliche zukünftige Verteilung der Weltbevölkerung darstellen

- Dynamik der Weltbevölkerung analysieren

- Ursachen und Auswirkungen der räumlichen und sozialen Mobilität in verschiedenen Gesellschaften vergleichen

Wesentlicher Bereich 4: Die wirtschaftlichen Bedürfnisse der Menschen

Die wirtschaftlichen Bedürfnisse der Menschen bewerten

- Bedeutung von Markt und Marktversagen erläutern

- Wirtschaftliche Ungleichheiten auf der Erde darstellen

- Ursachen wirtschafflicher Ungleichheiten beurteilen (politisches Handeln, Ressourcen, weltwirtschaftliche Strukturen)

- Die Produktion von Bedürfnissen hinsichtlich Konzepten der Nachhaltigkeit bewerten

Wesentlicher Bereich 5: Nutzungskonflikte an regionalen Beispielen

Nutzungskonflikte an regionalen Beispielen reflektieren

- Regionale Konflikte über die Verfügbarkeit von knappen Ressourcen (Boden, Wasser, Bodenschätze usw.) und dahinter stehende politische Interessen erklären

- Unterschiedliche Folgen von Naturereignissen aufgrund des sozialen und ökonomischen Gefüges beurteilen

- Tragfähigkeit der ,Einen Welt' zukunftsorientiert reflektieren 
Wesentlicher Bereich 1: Räumliche, soziale und ökonomische Entwicklungen in Europa

Raumbegriff und Strukturierung Europas reflektieren

- Gliederung Europas nach naturräumlichen, gesellschaftlichen und ökonomischen Merkmalen vergleichen

- Heterogene räumliche und ökonomische Auswirkungen des Integrationsprozesses der Europäischen Union untersuchen

- Europa-Konzepte kritisch reflektieren

Konvergenzen und Divergenzen europäischer Gesellschaften erörtern

- Gesellschaftliche und politische Entwicklungen im europäischen Kontext erläutern und deren Bedeutung für das eigene Leben hinterfragen

- Migrationen in und nach Europa erörtern

- Chancen der europäischen Bildungs- und Arbeitsmärkte für die eigene Lebens-und Berufsplanung erkennen

\section{Wesentlicher Bereich 2: Außerwert- und Inwertsetzung von Produktionsgebieten}

\section{Außerwert-und Inwertsetzung von Produktionsgebieten beurteilen}

- Abhängigkeit landwirtschaftlicher Nutzung vom Naturraumpotential untersuchen

- Strukturen und Wandel landwirtschaftlicher und industrieller Produktionsbedingungen in Europa vergleichen

- Eignung von Räumen für die Tourismusentwicklung sowie Folgen der Erschließung beurteilen

\section{Klasse (2. Semester)}

\section{Wesentlicher Bereich 1: Wettbewerbspolitik und Regionalpolitik}

\section{Wettbewerbspolitik und Regionalpolitik bewerten}

- Maßnahmen und Auswirkungen des europäischen Binnenmarktes erörtern

- Räumliche Disparitäten theoretisch begründen und anhand ausgewählter Beispiele veranschaulichen

- Träger, Instrumente, Funktionsweise und Ziele der Wettbewerbs- und Regionalpolitik erkennen und kritisch bewerten

\section{Wesentlicher Bereich 2: Regionale Entwicklungspfade}

\section{Regionale Entwicklungspfade vergleichen}

- Anhand ausgewählter Beispiele die Veränderungen in Raum, Wirtschaft und Gesellschaft durch Beitritt und Mitgliedschaft in der Europäischen Union erörtern

- Die Bedeutung grenzüberschreitender Zusammenarbeit für die Raumentwicklung erfassen

- (National)Staatlichkeit und Bildung neuer europäischer Regionen hinsichtlich ihrer Zukunftsfähigkeit reflektieren

\section{Klasse (1. Semester)}

\section{Wesentlicher Bereich 1: Wirtschaftsstandort Österreichs im europäischen und globalen Kontext}

Veränderungen der geopolitischen Lage Österreichs erläutern

- Qualitäten österreichischer Grenzen seit dem 20. Jahrhundert in ihren diversen Auswirkungen erläutern

- Möglichkeiten grenzüberschreitender Regionalentwicklung unter dem Einfluss der europäischen Integration darstellen

Wirtschaftsstandort Österreich beurteilen

- Vor- und Nachteile des Wirtschaftsstandorts Österreich aus unterschiedlicher Sicht erarbeiten und mit anderen Staaten vergleichen

- Entstehung regionaler Disparitäten analysieren

- Auswirkungen regionaler Disparitäten auf das Alltagsleben und die Wirtschaft erläutern

- Außenwirtschaft Österreichs in Zusammenhang mit europäischen und globalen Entwicklungen erörtern

- Lebensqualität in Österreich diskutieren

- WIKU: Industrie und Dienstleistung als wesentliche Basis der Wertschöpfung beschreiben und ihre jetzigen bzw. zukünftigen vernetzten Problemfelder beurteilen

Wesentlicher Bereich 2: Gesamtwirtschaftliche Leistungen und Probleme sowie Wirtschafts- und Sozialpolitik

Gesamtwirtschaftliche Leistungen und Probleme sowie Wirtschafts-und Sozialpolitik erklären

- Das Zustandekommen wirtschaftlicher Daten nachvollziehen und deren Aussagekraft beurteilen

- Besonderheiten der österreichischen Wirtschafts- und Sozialpolitik darstellen

- Gesamtwirtschaftliche Krisenmomente im Zusammenhang mit divergenten ökonomischen Theorien erklären

- Wirtschafts- und Sozialpolitik und ihre Zielkonflikte als interessensbezogen diskutieren und unterschiedliche Positionen argumentieren 


\section{Klasse (2. Semester)}

\section{Wesentlicher Bereich 1: Naturräumliche Chancen und Risiken}

Naturräumliche Chancen und Risiken erörtern

- Geoökologische Faktoren und Prozesse erklären

- Naturräumliche Gegebenheiten als Chance der Regionalentwicklung erkennen

- Naturräumliche sowie soziale Gegebenheiten und Prozesse als Ursachen ökologischer Probleme erörtern

- Eigene Strategien für ökologisch nachhaltiges Handeln entwickeln

Wesentlicher Bereich 2: Demographische Entwicklung und deren gesellschaftspolitische Implikationen

Demographische Entwicklung und gesellschaftspolitische Implikationen beurteilen

- Entwicklung der österreichischen Bevölkerung darstellen

- Mögliche soziale und ökonomische Folgen der Bevölkerungsentwicklung beurteilen

- Herausforderungen multikultureller und alternder Bevölkerungen erörtern

- Auswirkungen gesellschaftlicher Inklusions- und Exklusionsprozesse auf die Lebenssituationen ausgewählter Bevölkerungsgruppen bewerten

\section{Wesentlicher Bereich 3: Unternehmen und Berufsfelder}

Unternehmen und Berufsfelder analysieren

- Produkt- und Geschäftsideen für ein eigenes Unternehmen erstellen

- Schritte zu einer Unternehmensgründung beschreiben

- Grundzüge der Buchhaltung (Einnahmen- Ausgabenrechnung) herausarbeiten

- Eigene Möglichkeiten der Wahl von Bildungswegen und Beruf reflektieren

- WIKU: Betriebliche Kennzahlen interpretieren

- WIKU: Grundlagen betrieblichen Managements erfassen

\section{Klasse (1. Semester)}

Wesentlicher Bereich 1: Globalisierung gesellschaftlicher, politischer und ökonomischer Systeme

Chancen und Gefahren der Globalisierung erörtern

- Den eigenen Standort bzw. die lokale Betroffenheit in Prozessen der Globalisierung in Bezug auf persönliche Chancen und Risiken analysieren

- Globalen Wandel und seine ökonomischen, sozialen und ökologischen Ursachen und Wirkungen auch hinsichtlich der eigenen Lebenssituation - erörtern

- Auswirkungen ökonomischer Globalisierung diskutieren

- Strategien individuell, betrieblich und gesellschaftlich nachhaltigen Handelns entwickeln

- WIKU: Positionierungsmöglichkeiten von Unternehmen und Regionen in der globalisierten Wirtschaft analysieren

Politische und ökonomische Systeme vergleichen

- Ursachen und Auswirkungen sozialer und ökonomischer Disparitäten auf globaler Ebene beurteilen

- Formen, Möglichkeiten und Risiken der Entwicklungszusammenarbeit diskutieren

- Unterschiedliche Wirtschafts- und Regulierungsmodelle vergleichen

- Machtverhältnisse in politischen und ökonomischen Systemen analysieren

\section{Wesentlicher Bereich 2: Politische Gestaltung von Räumen}

Politische Gestaltung von Räumen untersuchen

- Ziele, Gestaltungsspielräume und Auswirkungen der Raumordnung erklären

- Konstruktionen von Räumen und raumbezogenen Identitäten untersuchen

- Bereitschaft entwickeln, zumindest auf der kommunalpolitischen Ebene gestaltend mitzuwirken

\section{Klasse (2. Semester)}

\section{Wesentlicher Bereich 1: Städte als Lebensräume und ökonomische Zentren}

Städte als Lebensräume und ökonomische Zentren untersuchen

- Vielfalt der subjektiven Wirklichkeiten in Städten vergleichen

- Soziale Differenzen in urbanen Räumen analysieren

- Prozesse von Urbanität und Urbanisierung beschreiben

- Bedeutung von Metropolen als Steuerungszentren der Wirtschaft beurteilen

- Perspektiven und Beispiele für eine nachhaltige Urbanität entwickeln

\section{Wesentlicher Bereich 2 (WIKU): Geld und Währung}

WIKU: Geld und Währung analysieren

- Entwicklungen in internationalen Kapitalströmen und Finanzmärkten analysieren Anlageformen nach Risiko und Chance bewerten 


\section{Acknowledgement}

Diese Publikation wurde aus Mitteln des Open-AccessPublikationsfonds der Universität Wien kofinanziert.

\section{Literatur}

BMB - Bundesministerium für Bildung (2016): Verordnung der Bundesministerin für Bildung, mit der die Verordnung über die Lehrpläne der allgemein bildenden höheren Schulen geändert wird; Bekanntmachung, mit der die Bekanntmachung der Lehrpläne für den Religionsunterricht an diesen Schulen geändert wird. Wien. BGBl. IINr. 219/2016 vom 9.8.2016, https://www.ris. bka.gv.at/eli/bgbl/II/2016/219 (20.7.2017).
BMB - Bundesministerium für Bildung (2017): Leistungsbeurteilung im Rahmen der NOST (LB NOST) Referenzpapier für LehrerInnenfortbildung (LFB) zum Thema "Wesentliche Bereiche des Lehrplans im Beiblatt des Semesterzeugnisses". Arbeitspapier.

Hinsch, S., H. Pichler, T. Jekel, L. Keller \& F. Baier (2014): Semestrierter Lehrplan AHS, Sekundarstufe II. Ergebnis der ministeriellen Arbeitsgruppe. In: GW-Unterricht 135. S. 51-61.

Jekel, T. \& H. Pichler (2017): Vom GW-Unterrichten zum Unterrichten mit geographischen und ökonomischen Konzepten. $\mathrm{Zu}$ den neuen Basiskonzepten im österreichischen GW-Lehrplan AHS Sek II. In: GW-Unterricht 147. S. $5-15$. 